\title{
Atom-Light Hybrid Interferometer
}

\author{
Bing Chen, ${ }^{1}$ Cheng Qiu, ${ }^{1}$ Shuying Chen, ${ }^{1}$ Jinxian Guo, ${ }^{1}$ L. Q. Chen, ${ }^{1, *}$ Z. Y. Ou, ${ }^{1,3, \dagger}$ and Weiping Zhang ${ }^{1,2, *}$ \\ ${ }^{1}$ Department of Physics, Quantum Institute for Light and Atoms, State Key Laboratory of Precision Spectroscopy, \\ East China Normal University, Shanghai 200062, People's Republic of China \\ ${ }^{2}$ Key Laboratory of Polar Materials and Devices, Ministry of Education, East China Normal University, Shanghai 200241, China \\ ${ }^{3}$ Department of Physics, Indiana University-Purdue University Indianapolis, \\ 402 North Blackford Street, Indianapolis, Indiana 46202, USA
}

(Received 1 January 2015; published 24 July 2015)

\begin{abstract}
A new type of hybrid atom-light interferometer is demonstrated with atomic Raman amplification processes replacing the beam splitting elements in a traditional interferometer. This nonconventional interferometer involves correlated optical and atomic waves in the two arms. The correlation between atoms and light developed with the Raman process makes this interferometer different from conventional interferometers with linear beam splitters. It is observed that the high-contrast interference fringes are sensitive to the optical phase via a path change as well as the atomic phase via a magnetic field change. This new atom-light correlated hybrid interferometer is a sensitive probe of the atomic internal state and should find wide applications in precision measurement and quantum control with atoms and photons.
\end{abstract}

Interferometers can be formed by coherent splitting and recombination of all kinds of waves ranging from optical waves [1] to de Broglie matter waves of electrons [2], neutrons [3], and even atoms and molecules [4]. They are widely used in precision measurement of a variety of physical quantities. Building on this foundation, nonconventional interferometers can be constructed with nonlinear processes such as wave splitting and recombination elements [5-10], as shown in the inset of Fig. 1.

Different from the conventional interferometers with beam splitters, the involvement of nonlinear processes in the nonconventional interferometers allows the coupling between two waves of different types, and it can lead to interference fringes that are sensitive to different types of phase shifts. We thus use the word "hybrid" to label these interferometers involving different types of waves. In fact, hybrid interference also occurs via coherent interactions between atoms and light in phenomena such as quantum storage in electromagnetically induced transparency [11-13], gradient echo memory [14,15], and slow light [16-20]. However, the hybrid interference effects in these phenomena are in essence still of the same type as the conventional interference effect where the input wave is linearly split into a linear superposition of atom and light fields in the form of a polariton state $[11,15]$. On the other hand, a nonconventional SU(1,1) interferometer $[5,9,10,21]$ utilizes parametric amplifiers as wave splitting and recombination elements and performs quite differently from the conventional linear interferometers. The name SU(1,1) comes from the nonlinear interaction Hamiltonian for the parametric process [5]:

$$
\hat{H}_{\mathrm{SU}(1,1)}=i \hbar \eta \hat{a}_{s}^{\dagger} \hat{a}_{i}^{\dagger}+\text { H.c., }
$$

which amplifies an input signal field $\left(\hat{a}_{s}\right)$ and produces a correlated idler field $\left(\hat{a}_{i}\right)$ simultaneously. The idler field is coherent with the input field, thus realizing coherent wave splitting.

One of the nonlinear processes described by Eq. (1) is the collective atomic Raman amplification process, which

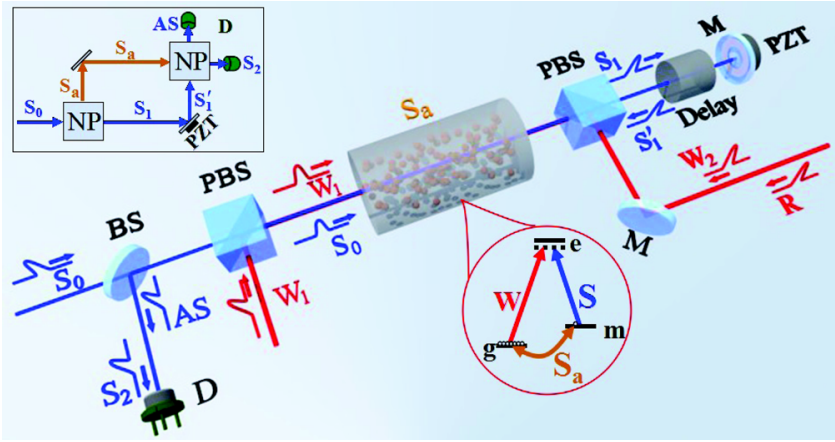

FIG. 1 (color online). Experimental sketch for the hybrid atomlight interferometer. A strong Raman write beam $\left(W_{1}\right.$, red) and a Stokes input field $\left(S_{0}\right.$, blue) in orthogonal polarization interact with a $\Lambda$-shaped atomic system to generate an amplified Stokes field $\left(S_{1}\right)$ and a correlated atomic spin wave $S_{a}$ that stays in the atomic system. The amplified Stokes beam $\left(S_{1}\right)$, after reflection $\left(S_{1}^{\prime}\right)$, is sent back together with another strong write beam $\left(W_{2}\right)$ to the atomic cell after some optical delay to recombine with the waiting atomic spin wave $S_{a}$ for superposition. The final Stokes field $\left(S_{2}\right)$ (generated by $W_{2}$ and $S_{1}^{\prime}$ ) is detected by $D$, together with a delayed anti-Stokes field (AS) due to a strong delayed reading beam $\left(R\right.$, red) for the atomic spin wave readout. Both $S_{2}$ and AS show interference fringes. Top inset: an interferometer with nonlinear processes (NP) for wave splitting and recombination. The labels correspond to the main figure. Bottom inset: atomic energy levels. 
involves an optical field (Stokes) and a correlated atomic spin wave. In this Letter, we report on the construction and the study of a brand new type of hybrid atom-light correlated interferometer which uses the collective Raman amplification processes to generate the correlated optical and atomic waves and then recombine the waves for interference. In-phase interference fringes are observed for the optical and atomic outputs as we scan either the optical phase or the atomic phase. Although our early versions of interferometers reported in Refs. $[9,21]$ also involve the SU(1,1) interaction in Eq. (1), they are alloptical types relying on photon-photon correlations and can only measure optical phases. The interferometer reported here goes one step further to have atoms actively involved, leading to atom-photon correlations via $\mathrm{SU}(1,1)$ interaction. Thus, the interference fringes that result from atomphoton correlations depend on both atomic and optical phases so that we can probe the atomic phases with optical interferometric techniques. Furthermore, as demonstrated in Ref. [21], this interferometer should be able to work with a phase measurement precision beyond the standard quantum limit.

The schematic diagram of the atom-light interferometer is shown in Fig. 1. When an ensemble of $N_{a}$ atoms with a pair of lower metastable states $|g\rangle,|m\rangle$ (shown in the inset of Fig. 1) is pumped by a strong Raman write field $\left(W_{1}\right)$, a collective Raman process acts as a Raman amplifier for an input Stokes field $\left(S_{0}\right)$ and generates a collective atomic excitation field (also known as atomic pseudospin wave) $\hat{S}_{a} \equiv\left(1 / \sqrt{N_{a}}\right) \sum_{k}|g\rangle_{k}\langle m|$ that is correlated to the amplified Stokes field $\left(S_{1}\right)$. The three waves, i.e., the strong Raman write field $\left(A_{W}\right)$, the Stokes field $\left(\hat{a}_{S}\right)$, and the atomic spin wave $\left(\hat{S}_{a}\right)$ are coupled via an upper excited level $|e\rangle$, which can be adiabatically eliminated leading to a Hamiltonian given by $[22,23]$

$$
\hat{H}_{R}=i \hbar \eta A_{W} \hat{a}_{S}^{\dagger} \hat{S}_{a}^{\dagger}-i \hbar \eta^{*} A_{W}^{*} \hat{a}_{S} \hat{S}_{a},
$$

where $\eta=g_{e g} g_{e m} / \Delta$ with $g_{e g}, g_{e m}$ as the coupling coefficients between the excited state and the lower level states. $\Delta$ is the detuning from the excited state for both the Stokes and Raman write fields, which satisfy the two-photon resonance condition: $\omega_{W}-\omega_{S}=\omega_{m g}$. When the write field $A_{W}$ is relatively weak, the collective Raman scattering is in spontaneous mode and can generate correlated atomic excitations and single photons, which has been widely used in quantum memory [24-28]. When the write field $A_{W}$ is relatively strong, the collective Raman scattering is in the stimulated mode and can amplify the input field to produce intensity and phase correlated atom-light fields $[29,30]$.

Notice that the Hamiltonian in Eq. (2) has exactly the same form as the SU(1,1) Hamiltonian in Eq. (1) for the parametric process. Here, the Stokes field is equivalent to the signal field and the atomic spin wave is the idler field. So, the parametric amplifiers in the nonconventional
$\mathrm{SU}(1,1)$ interferometer $[5,9,10,21]$ can be replaced with Raman amplifiers to form an atom-light hybrid interferometer. Different from the all-optical versions $[9,21]$, the atomic spin wave $S_{a}$ stays in the atomic cell while the Stokes field $S_{1}$ travels out of the atomic cell. So, to recombine the Stokes field $S_{1}$ and the atomic spin wave $S_{a}$ for interference, we send the Stokes field back into the atomic cell (marked as $S_{1}^{\prime}$ ) together with another strong write field $W_{2}$ after some delay, as shown in Fig. 1. The delay is necessary so as to temporally separate the wave splitting and recombination processes because we are using the same atomic cell for the two processes. Linearly polarized write field and Stokes field have orthogonal polarizations due to atomic transition selection rules. A polarization beam splitter (PBS) is used to separate them so that optical phase changes can be applied only to the Stokes field. To observe the interference fringe, the output Stokes field $S_{2}$ is monitored by detector $D$. In the meantime, we can also observe the final value of the atomic spin wave $S_{a}$ in the atomic ensemble by a delayed reading beam $R$, which converts $S_{a}$ into an anti-Stokes field (AS). In our experiment, the conversion efficiency is around $35 \%$. The antiStokes field is delayed and can be also observed in detector $D$. This is equivalent to observing the output at the idler field in the all-optical version [9].

The interference fringes shown in Fig. 2 are measured when we scan the optical phase of the $S_{1}^{\prime}$ field by moving mirror $\mathrm{M}$ with a piezoelectric transducer (PZT) (as shown in Fig. 1). The two fringes correspond to two different initial input states: Fig. 2(a) is for the initial input at the Stokes field, shown as $S_{0}$ in Fig. 1, while Fig. 2(b) is for an initially prebuilt atomic spin wave $S_{a_{0}}$ as the input field (not shown in Fig. 1), but without $S_{0}$. The initial atomic spin
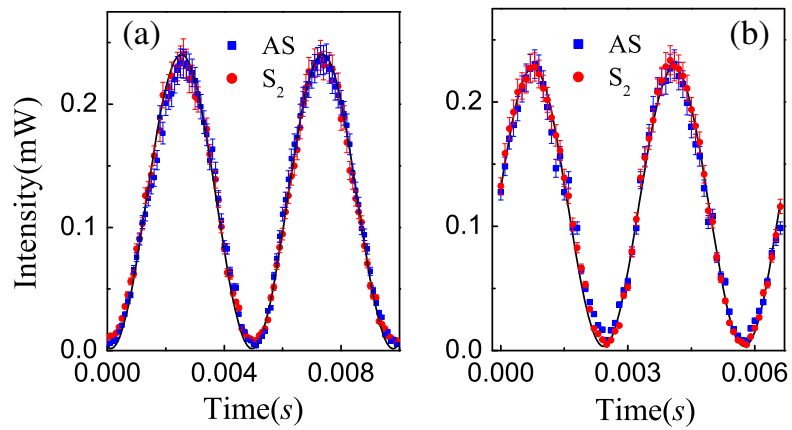

FIG. 2 (color online). Observed interference fringes in two output signals ( $S_{2}$ and AS) as the optical phase is scanned. (a) The input field is the light field $S_{0}$. The Stokes light output has a slightly smaller visibility (94\%) than the readout anti-Stokes $(96.5 \%)$. (b) The input field is the atomic spin wave $\left(S_{a 0}\right)$ (see the Supplemental Material [31] for this arrangement). The Stokes light output has a slightly higher visibility $(96.3 \%)$ than the antiStokes readout (93.6\%). In both figures, the red circles are for the Stokes light output and the blue squares are for the anti-Stokes light output (2.9 times magnified for comparison with the Stokes), which represents the atomic spin wave. 
wave $S_{a_{0}}$ is prebuilt by another Raman process (not shown, see Supplemental Material [31] for details). Figure 2(b) is equivalent to an initial input at the idler port for the all-optical SU(1,1) interferometer [9]. In Fig. 2(a), the visibility of $S_{2}$ is $94.0 \%$, a little smaller than that of AS at 96.5\%. In Fig. 2(b), because the initial atomic spin wave is nonzero instead of the Stokes field as in Fig. 2(a), the roles of the Stokes field and the atomic spin wave (as exhibited in the anti-Stokes field) are switched: the visibility of AS is $93.6 \%$, a little smaller than that of $S_{2}$ at $96.3 \%$. The lesser visibilities are due to the initial input as a background, which cannot be canceled even with complete destructive interference [9]. Notice that the interference fringes in Fig. 2 are in phase for the two outputs (Stokes and antiStokes) of the interferometer. This is in contrast to the fringes that are 180 degree out of phase in the two outputs of a conventional linear interferometer.

Different from the all-optical SU $(1,1)$ interferometers in Refs. [9,21], atomic spin waves are involved in this interference scheme and the interference fringes should depend on the phase of the atomic spin wave, which can be changed by an external field such as the magnetic field. As is well known, the atomic level $j$, when subject to a $B$ field, is shifted due to the Zeeman effect by $\Delta \omega_{j}=m_{j} g_{j} \mu_{B} B / \hbar$, where $\mu_{B}$ is the Bohr magnetic moment, $g_{j}$ is the Landé factor for energy level $j$, and $m_{j}$ is the magnetic quantum number of the Zeeman sublevel. Therefore, after a time interval $T$, the atomic spin wave $\hat{S}_{a}=\left(1 / \sqrt{N_{a}}\right) \sum_{k}|g\rangle_{k}\langle m|$ will evolve to $\hat{S}_{a}^{\prime}=\left(1 / \sqrt{N_{a}}\right) \sum_{k} e^{i \Delta \omega_{g} T}|g\rangle_{k}\langle m| e^{-i \Delta \omega_{m} T}=$ $\hat{S}_{a} e^{-i \varphi_{a}}$, where the atomic phase shift is

$$
\varphi_{a}=\left(m_{j_{m}} g_{m}-m_{j_{g}} g_{g}\right) \mu_{B} B T / \hbar=\gamma B T,
$$

with $\gamma \equiv\left(m_{j_{m}} g_{m}-m_{j_{g}} g_{g}\right) \mu_{B} / \hbar$. Thus, the atomic phase is directly proportional to the applied magnetic field $B$ and the optical delay $T$. In the experiment, the external magnetic field $B$ comes from a helix coil around the atomic cell inside the magnetic shielding. The magnetic field is parallel to the propagation direction of the light fields. Unfortunately, the ground state $|g\rangle$ and the metastable state $|m\rangle$ have many Zeeman sublevels. When the $B$ field is applied, the Zeeman shifts of each sublevel are different. So, we must choose one Zeeman sublevel from each of the states $|g\rangle$ and $|m\rangle$ so that there is only one atomic spin wave to interact with the optical fields. To achieve this, we use circularly polarized optical fields: $\sigma+$ for the Raman write beam and $\sigma-$ for the Stokes beam. However, these two circularly polarized optical fields are still coupled to two atomic spin waves: $\hat{S}_{a}^{(1)}=\left|5 S_{1 / 2}, F=1, m_{F}=0\right\rangle$ $\left\langle 5 S_{1 / 2}, F=2, m_{F}=2\right|$, and $\hat{S}_{a}^{(2)}=\mid 5 S_{1 / 2}, F=1, m_{F}=$ $-1\rangle\left\langle 5 S_{1 / 2}, F=2, m_{F}=1\right|$ [see the energy level diagram in Fig. 3(b)]. We can restrict the involvement of $\hat{S}_{a}^{(2)}$ by preparing an initial atomic spin wave in $\hat{S}_{a}^{(1)}$ only (see

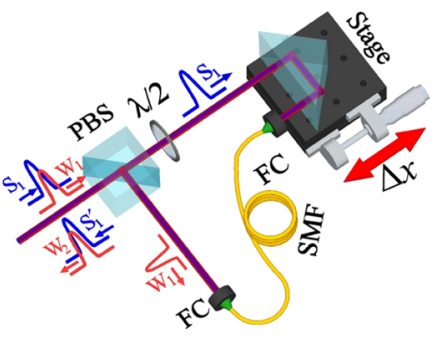

(a)

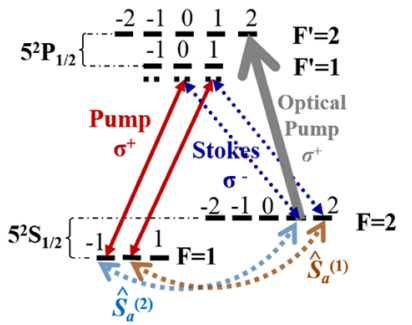

(b)
FIG. 3 (color online). Sagnac loop for the optical delay. (a) We use the Sagnac configuration for the write and Stokes delay to stabilize the optical phase of the interferometer: The write and Stokes fields travel in the opposite directions inside the Sagnac loop to cancel any optical phase change due to mirror vibration. Red: Raman write field; blue: Stokes field; $\lambda / 2$ : half wave plate to rotate the polarization angles by 90 degrees; FC: fiber coupler; SMF: single-mode fiber; Stage: linear translational stage. (b) Magnetic sublevels for optical and multiple atomic spin waves.

Supplemental Material [31] for details). Without a prebuilt atomic spin wave in $\hat{S}_{a}^{(2)}$, there will be only spontaneous Raman scattering involving $\hat{S}_{a}^{(2)}$, which is much weaker than the enhanced Raman scattering by the initially prepared atomic spin wave in $\hat{S}_{a}^{(1)}$ [33].

Since the interference fringe is sensitive to both optical and atomic phases in this interferometer, in order to reveal the interference fringe by atomic phase scan, we need to stabilize the optical phase. However, this is not easy because any shaking of the mirrors leads to random optical phase change, resulting in the instability of the interference fringe. Here, we use a Sagnac configuration as shown in Fig. 3(a) to stabilize the optical phase. In this configuration, the write field and the Stokes field coming out of the atomic cell after the first Raman interaction travel in the same path but in the opposite directions inside a Sagnac loop before being combined and sent back to the cell for the second parametric interaction. In this way, the optical phase instability due to mirror shaking is canceled because the fringe depends on $\varphi_{W}-\varphi_{S}$.

With the optical phase stabilized, we can change the atomic phase by linearly ramping up the magnetic field with a controlled current source. The interference fringes are shown in Fig. 4 for three different optical delays. The visibilities are all around $96 \%$. The solid curves are the best fits to the sine functions. From the best fits, we may find the periods of each sine function and we plot them in Fig. 4(d) as a function of the optical delay length. A linear dependence is expected from what we discussed above about the atomic phase. The slope of the linear fit is $0.0375 \mathrm{rad} /(\mathrm{G} \cdot \mathrm{m})$.

Even with the Sagnac configuration, we can still change optical phase with a different method. Because the Raman write field and the Stokes field have a $6.87 \mathrm{GHz}$ frequency difference (or $\Delta k=142 \mathrm{rad} / \mathrm{m}$ ), there is actually an extra optical phase difference of 

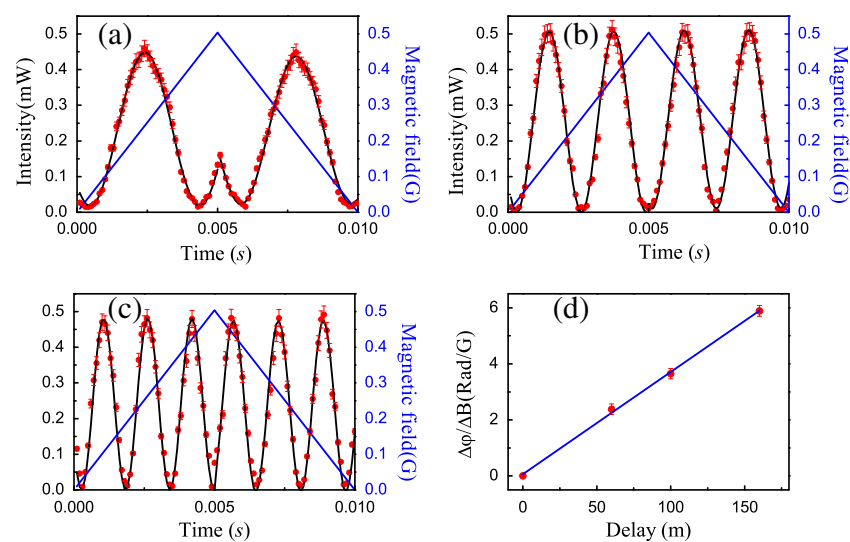

FIG. 4 (color online). Interference fringes by scanning the atomic phase via magnetic field. Optical delay is (a) 60, (b) 100, and (c) $160 \mathrm{~m}$, respectively. Red dot: the real interference fringe; black line: the fitting curve of the interference fringe; blue line: the ramp scan of the magnetic field. (d) The phase sensitivity with respect to the magnetic field change as a function of the fiber delay length.

$$
\Delta \theta_{\mathrm{op}}=\varphi_{W}-\varphi_{S}=\Delta k \cdot \Delta s \approx 284(\mathrm{rad} / \mathrm{m}) \Delta x(\mathrm{~m}) .
$$

Here, $\Delta s$ is the optical path change and $\Delta x \equiv \Delta s / 2$ is the displacement of the mirror mounted on a translational stage, as shown in Fig. 3. This optical phase difference shows up as a shift in the interference fringe of the magnetic field scan, as shown in Fig. 5(a). From the fit to the sine function, we can extract the phase shift, which is plotted in Fig. 5(b) as a function of $\Delta x$. We find from Fig. 5(b) that $\Delta x=21 \pm 1 \mathrm{~mm}$ corresponds to a phase shift of $2 \pi$, in agreement with the predicted value from Eq. (4).

The dependance of the output fringe phase on the magnetic field suggests that this interferometer can be used as a magnetometer, which operates on a different scheme for atomic phase readout from traditional atomic magnetometers $[34,35]$. The sensitivity of this new magnetometer depends on the noise performance of the SU(1,1) interferometer. From Refs. $[10,21]$, we learned that the phase measurement sensitivity of this type of interferometer is in principle better than the standard quantum limit or the shot noise limit. Thus, the magnetometer based on this interferometer can potentially beat the atomic projection quantum noise in a traditional atomic magnetometer and promises a better sensitivity.

As shown in Ref. [10], the sensitivity of the $\mathrm{SU}(1,1)$ nonconventional interferometer is limited by losses inside the interferometer. The underlying physics is that the losses in both arms of the interferometer destroy partially the quantum correlation that is crucial in making the output noise level low. Moreover, the uncorrelated vacuum noise that is leaked through via the losses is further amplified by the second parametric (Raman) amplifier and will eventually add to the output and reduce the sensitivity. However, for our atom-light interferometer, loss in the atomic spin wave is due to decoherence, which is in the form of an
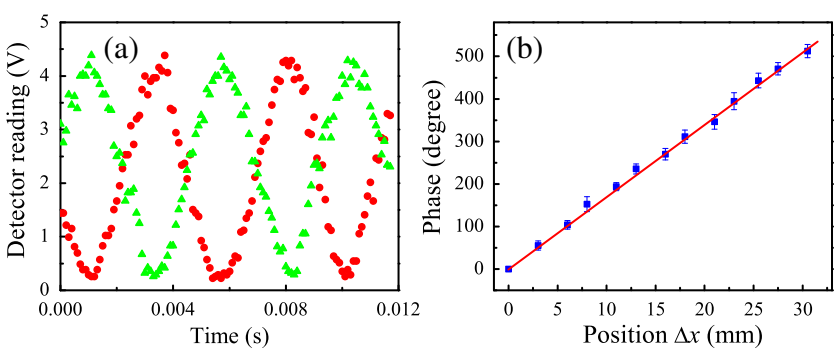

FIG. 5 (color online). Optical phase shift as a function of the stage displacement. (a) Interference fringes as the atomic phase is scanned via the magnetic field at two different locations of the translational stage. (b) Relative phase shifts derived from (a) as a function of the position of the translational stage. The solid red line is a linear fit. The fit slope is $16.9 \pm 0.7$ degree $/ \mathrm{mm}$ and in accordance with the theoretical value of 16.3 degree $/ \mathrm{mm}$ found via Eq. (4).

exponential decay $e^{-t / T_{C}}$, where $T_{C}$ is the decoherence time that mostly depends on collisions with the cell walls. So the loss can be made very small with a short interaction time or large decoherence time $T_{C}$ by buffer gases or antirelaxation coatings [36]. Hence, most of the loss comes from the Stokes field. In this situation, the amplified vacuum noise leaked through the lossy channel can be canceled due to the quantum entanglement nature of the parametric amplifier and output noise level of the interferometer is basically the same as the ideal lossless case [10,32] (see detail in the Supplemental Material [31]). Thus the improvement in sensitivity over the shot noise limit can be quite large. This is the advantage of involving atomic states, which are basically lossless.

The atomic phase is not only sensitive to magnetic fields, it can also be changed by ac Stark shifts due to the illumination of another nonresonant optical field: $\varphi_{a}=$ $\Delta \omega_{\mathrm{ac}} T$ with $\Delta \omega_{\mathrm{ac}}$ as the ac Stark shift $[37,38]$ and $T$ as the illumination time. $\Delta \omega_{\mathrm{ac}}$ is proportional to the intensity of the illuminating nonresonant field. This can lead to nondestructive measurement of the intensity of an optical field.

In summary, this atom-light interferometer will open a new door for precision measurements and for probing and controlling atomic states.

This work was supported by the National Basic Research Program of China (973 Program Grant No. 2011CB921604), the National Natural Science Foundation of China (Grants No. 11274118, No. 11129402, No. 11234003, and No. 91436211-2), and supported by Innovation Program of Shanghai Municipal Education Commission (Grant No. 13ZZ036), the fundamental research funds for the central universities.

\footnotetext{
*lqchen@phy.ecnu.edu.cn

tzou@iupui.edu

+wpzhang@phy.ecnu.edu.cn
} 
[1] M. Born and E. Wolf, Principles of Optics 1st ed. (Pergamon, Oxford, 1959); Principles of Optics, 7th ed. (Pergamon, Oxford, 1999).

[2] L. Marton, J. Arol Simpson, and J. A. Suddeth, Phys. Rev. 90, 490 (1953); G. Möllenstedt and H. Düker, Naturwissenschaften 42, 41 (1955).

[3] H. Rauch, W. Treimer, and U. Bonse, Phys. Lett. 47A, 369 (1974).

[4] A. D. Cronin, J. Schmiedmayer, and D. E. Pritchard, Rev. Mod. Phys. 81, 1051 (2009).

[5] B. Yurke, S. L. McCall, and J. R. Klauder, Phys. Rev. A 33, 4033 (1986).

[6] Z. Y. Ou, Phys. Rev. A 55, 2598 (1997).

[7] D. Leibfried, B. DeMarco, V. Meyer, M. Rowe, A. Ben-Kish, J. Britton, W. M. Itano, B. Jelenković, C. Langer, T. Rosenband, and D. J. Wineland, Phys. Rev. Lett. 89, 247901 (2002).

[8] W. N. Plick, J. P. Dowling, and G. S. Agarwal, New J. Phys. 12, 083014 (2010).

[9] J. Jing, C. Liu, Z. Zhou, Z. Y. Ou, and W. Zhang, Appl. Phys. Lett. 99, 011110 (2011).

[10] Z. Y. Ou, Phys. Rev. A 85, 023815 (2012).

[11] M. Fleischhauer and M. D. Lukin, Phys. Rev. Lett. 84, 5094 (2000).

[12] D. F. Phillips, A. Fleischhauer, A. Mair, R. L. Walsworth, and M. D. Lukin, Phys. Rev. Lett. 86, 783 (2001).

[13] A. Mair, J. Hager, D. F. Phillips, R. L. Walsworth, and M. D. Lukin, Phys. Rev. A 65, 031802(R) (2002).

[14] M. Hosseini, B. M. Sparkes, G. Campbell, P. K. Lam, and B. C. Buchler, Nat. Commun. 2, 174 (2011).

[15] G. Campbell, M. Hosseini, B. M. Sparkes, P. K. Lam, and B. C. Buchler, New J. Phys. 14, 033022 (2012).

[16] A. S. Zibrov, A. B. Matsko, O. Kocharovskaya, Y. V. Rostovtsev, G. R. Welch, and M. O. Scully, Phys. Rev. Lett. 88, 103601 (2002).

[17] J. J. Longdell, E. Fraval, M. J. Sellars, and N. B. Manson, Phys. Rev. Lett. 95, 063601 (2005).

[18] M. F. Yanik and S. Fan, Phys. Rev. A 71, 013803 (2005).

[19] R. M. Camacho, P. K. Vudyasetu, and J. C. Howell, Nat. Photonics 3, 103 (2009).

[20] G. Heinze, C. Hubrich, and T. Halfmann, Phys. Rev. Lett. 111, 033601 (2013).
[21] F. Hudlist, J. Kong, C. Liu, J. Jing, Z. Y. Ou, and W. Zhang, Nat. Commun. 5, 3049 (2014).

[22] L. M. Duan, M. D. Lukin, J. I. Cirac, and P. Zoller, Nature (London) 414, 413 (2001).

[23] K. Hammerer, A. S. Sorensen, and E. S. Polzik, Rev. Mod. Phys. 82, 1041 (2010).

[24] C. W. Chou, H. de Riedmatten, D. Felinto, S. V. Polaykov, S. J. van Enk, and H. J. Kimble, Nature (London) 438, 828 (2005).

[25] T. Chanelière, D. N. Matsukevich, S. D. Jenkins, S. Y. Lan, T. A. B. Kennedy and A. Kuzmich, Nature (London) 438, 833 (2005).

[26] B. Zhao, Z.-B. Chen, Y.-A. Chen, J. Schmiedmayer, and J.-W. Pan, Phys. Rev. Lett. 98, 240502 (2007).

[27] Z.-S. Yuan, Y.-A. Chen, B. Zhao, S. Chen, J. Schmiedmayer, and J.-W. Pan, Nature (London) 454, 1098 (2008).

[28] M. Bashkansky, F. K. Fatemi, and I. Vurgaftman, Opt. Lett. 37, 142 (2012).

[29] C.-L. Bian, L.-Q. Chen, G.-W. Zhang, Z. Y. Ou, and W. Zhang, Europhys. Lett. 97, 34005 (2012).

[30] M. G. Raymer, J. Mod. Opt. 51, 1739 (2004).

[31] See Supplemental Material at http://link.aps.org/ supplemental/10.1103/PhysRevLett.115.043602 for description of the detailed experimental arrangement and preparation of the initial atomic spin wave. Theoretical calculations are discussed in detail for immunity to optical loss in atom-light interferometer, which includes Refs. [10,21,32].

[32] J. Kong, F. Hudelist, Z. Y. Ou, and W. Zhang, Phys. Rev. Lett. 111, 033608 (2013).

[33] L. Q. Chen, G.-W. Zhang, C.-H. Yuan, J. Jing, Z. Y. Ou, and W. Zhang, Appl. Phys. Lett. 95, 041115 (2009).

[34] D. Budker and M. V. Romalis, Nat. Phys. 3, 227 (2007).

[35] A. Ben-Kish and M. V. Romalis, Phys. Rev. Lett. 105, 193601 (2010).

[36] M. V. Balabas, T. Karaulanov, M. P. Ledbetter, and D. Budker, Phys. Rev. Lett. 105, 070801 (2010).

[37] M. Brune, S. Haroche, V. Lefevre, J. M. Raimond, and N. Zagury, Phys. Rev. Lett. 65, 976 (1990).

[38] N. A. Proite and D. D. Yavuz, Opt. Commun. 282, 3275 (2009). 